\title{
The shape of the Red Giant Branch Bump as a diagnostic of partial mixing processes in low-mass stars
}

\author{
Santi Cassisi \\ Osservatorio Astronomico di Collurania, via M. Maggini, 64100 Teramo, Italy; \\ cassisi@te.astro.it. \\ Maurizio Salaris \\ Astrophysics Research Institute, Liverpool John Moores University, Twelve Quays House, \\ Egerton Wharf, Birkenhead CH41 1LD, United Kingdom; ms@astro.livjm.ac.uk. \\ Osservatorio Astronomico di Collurania, via M. Maggini, 64100 Teramo, Italy. \\ Max Planck Institut für Astrophysik, Karl-Schwarzschild-Str. 1, 85748 Garching, Germany. \\ Giuseppe Bono \\ Osservatorio Astronomico di Roma, Via Frascati 33, 00040 Monte Porzio Catone, Italy; \\ bono@coma.mporzio.astro.it.
}




\begin{abstract}
We suggest to use the shape of the Red Giant Branch (RGB) Bump in metal-rich globular clusters as a diagnostic of partial mixing processes between the base of the convective envelope and the H-burning shell. The Bump located along the differential luminosity function of cluster RGB stars is a key observable to constrain the H-profile inside these structures. In fact, standard evolutionary models that account for complete mixing in the convective unstable layers and radiative equilibrium in the innermost regions do predict that the first dredge-up lefts over a very sharp H-discontinuity at the bottom of the convective region.

Interestingly enough we found that both atomic diffusion and a moderate convective overshooting at the base of the convective region marginally affects the shape of the RGB Bump in the differential Luminosity Function (LF). As a consequence, we performed several numerical experiments to estimate whether plausible assumptions concerning the smoothing of the H-discontinuity, due to the possible occurrence of extra-mixing below the convective boundary, affects the shape of the RGB Bump. We found that the difference between the shape of RGB Bump predicted by standard and by smoothed models can be detected if the H-discontinuity is smoothed over an envelope region whose thickness is equal or larger than 0.5 pressure scale heights. Finally, we briefly discuss the comparison between theoretical predictions and empirical data in metal-rich, reddening free Galactic Globular Clusters (GGCs) to constrain the sharpness of the H-profile inside RGB stars.
\end{abstract}

Subject headings: globular clusters: general - stars: evolution - interiors Population II 


\section{Introduction}

One of the most intriguing features of GGCs is the occurrence of a local maximum in the distribution of RGB stars. It appears as a Bump in the differential LF, and as a change of slope in the cumulative LF. After the pioneering investigations by Thomas (1967) and Iben (1968), it is well known that this feature is due to the fact that during the RGB evolution the H-burning shell crosses the sharp chemical discontinuity left over by the convective envelope at the base of the RGB during its maximum sinking (first dredge-up). The abrupt change in the hydrogen abundance affects the efficiency of the H-burning shell, since the stellar structure adjusts to the increase in the available fuel, and the stellar luminosity undergoes a temporary drop. Dating back to the first detection in the GGC 47 Tucanae (King, Da Costa \& Demarque 1985), the RGB Bump has been the crossroad of several theoretical and observational investigations (Fusi-Pecci et al. 1990; Alongi et al. 1991; Bono \& Castellani 1992; Alves \& Sarajedini 1999; Ferraro et al. 1999; Zoccali et al. 1999; Bergbusch \& Vandenberg 2001, and reference therein). The main reason for this effort is that the RGB Bump is a key observable to investigate the chemical profile inside RGB structures, and a robust diagnostic of the maximum depth reached by the outer convection during the first dredge-up.

Till a few years ago a quantitative comparison between theory and observations was mainly hampered by the size of the available stellar samples along the RGB. This problem is particularly severe for the most metal-poor GGCs, since the RGB evolutionary timescales are shorter than in metal-rich ones. Moreover, the RGB Bump in metal-poor clusters is located at brighter magnitudes, and therefore in a RGB region poorly populated when compared with metal-rich clusters. However, the Hubble Space Telescope (HST) with its superior imaging quality and spatial resolution allowed us to firmly detect this feature in a sample of GGCs that cover a wide metallicity range (Zoccali et al. 1999).

This paper is the fifth in a series devoted to the RGB Bump in GGCs. Cassisi \& Salaris (1997) investigated the dependence of the Bump luminosity on the physical inputs adopted to construct stellar models. They showed that the difference in magnitude between the RGB Bump and the horizontal branch (HB) at the RR Lyrae instability strip $\left(\Delta V_{B u m p}^{H B}\right)$ predicted by their updated evolutionary models agrees quite well with empirical estimates of GGCs for which are available accurate spectroscopic measurements of cluster heavy element abundances. This evidence was further strengthened by the finding that $\Delta V_{B u m p}^{H B}$ is only marginally affected by atomic diffusion (Cassisi, Degl'Innocenti \& Salaris 1997). A thorough comparison between predicted and observed $\Delta V_{B u m p}^{H B}$ values brought forth that theory and observations do agree at the level of $\approx 0.1$ mag (Zoccali et al. 1999). To assess on a more quantitative basis the accuracy of current evolutionary models, Bono et al. 
(2001) compared the evolutionary lifetimes during the crossing of the H-discontinuity with the star counts across the RGB Bump. It turned out that theory is in very good agreement with observations over a wide metallicity range. Only a few clusters were at odds with theoretical predictions and 47 Tuc is one of them.

These investigations support the following evidence: i) the maximum depth reached by the convective envelope at the base of the RGB, whose diagnostic is the $\Delta V_{B u m p}^{H B}$ parameter, is correctly predicted by stellar models. However, the parameter $\Delta V_{B u m p}^{H B}$ relies on the distances provided by HB models. As a consequence, the agreement between theory and observations heavily relies on the adopted HB distance scale as well as on the GGC metallicity scales (Rutledge, Hesser \& Stetson 1997; Zoccali \& Piotto 2000; Bergbusch \& Vandenberg 2001); ii) the extent of the H-discontinuity left over by the convective envelope, whose diagnostic is the star counts across the RGB Bump, does depend neither on the distance scale, nor on the metallicity scale and it is correctly predicted by theory (Bono et al. 2001).

The main aim of this investigation is to study in detail how the sharpness of the H-discontinuity affects the RGB Bump. In particular, we are interested in estimating how this jump in the chemical composition affects the shape of the Bump in the differential LF. The reason for such an investigation is twofold: i) current theoretical models do not firmly predict the efficiency and extent of all possible mixing processes occurring in stellar interiors as well as the occurrence of any partial mixing below the formal boundary of the convective region. It is worth mentioning that these physical mechanisms are no more pure speculative problems. In fact, Gratton et al. (2001) collected with VLT/UVES high dispersion spectra for a sizable sample of stars in two GGCs and found that atomic diffusion presents a very low efficiency when moving from MS to SGB stars (see also Thévenin et al. 2001). This empirical evidence, once confirmed by new data, can supply useful hints on the occurrence of extra-mixing processes in the region located below the convective envelope. ii) The sharpness of the H-discontinuity does affect the evolutionary timescales (Bono \& Castellani 1992) of the H-burning shell during the crossing of the H-discontinuity, and in turn the shape of the Bump. Therefore a change in the extent of the smoothing region causes a change in the shape that can be constrained on the basis of the comparison with empirical data in metal-rich GGCs.

In $\S 2$ we briefly discuss the stellar models adopted in our investigation, while in $\S 3$ we investigate how different assumptions on the smoothing lengths affect the shape of the Bump and the star counts across the Bump. This analysis is performed by using a Monte-Carlo technique. In this section we also discuss what are the observational requirements and the target that can allow us to detect a change in the shape of the Bump. Conclusions and 
future developments are outlined in $\S 4$.

\section{Stellar models}

The evolutionary code and input physics adopted in this investigation are the same as in Cassisi \& Salaris (1997). Theoretical models were transformed into the observational plane by adopting the bolometric corrections and color-temperature relations provided by Castelli, Gratton \& Kurucz (1997a,b). We have focused our attention on a chemical composition typical of metal-rich GGCs, namely $\mathrm{Y}=0.23$ and $\mathrm{Z}=0.006$. Note that, if we account for an $\alpha$-enhancement of $[\alpha / \mathrm{Fe}]=0.4$, this metallicity implies $[\mathrm{Fe} / \mathrm{H}]=-0.8$, i.e. the metallicity at which the number of RGB stars is expected to be the largest among the sample of GGCs. The numerical experiments were also performed at fixed stellar mass $M / M_{\odot}=1$, since along the RGB the value of the evolving mass is almost constant. However, our conclusions do not depend on the selected mass value.

We computed several series of evolutionary models, from the Zero Age Main Sequence up to the RGB phases brighter than the Bump region. These models were constructed by neglecting or by accounting for atomic diffusion, and for various overshooting efficiencies namely $0.1-0.2 H_{p}$, where $H_{p}$ is the pressure scale height at the base of the convective envelope. According to Alongi et al. (1991) stellar models which include convective overshooting were constructed by assuming instantaneous mixing in the overshooting region. These numerical experiments were performed to estimate whether these two physical mechanisms affect the sharpness of the H-profile. Interestingly enough, we found that in all these models the H-discontinuity is sharp, and the shape of the Bump in the differential LF is the same as in standard models. This finding suggests that any detection of a peculiar shape in a cluster RGB Bump is almost certainly caused by a smoothing of the H-discontinuity.

As a consequence, we performed the experiments on our standard models, i.e. the evolutionary models that neglect both atomic diffusion and convective overshooting, and at the base of the RGB we artificially modified, according to Bono \& Castellani (1992), the abundance profiles below the point of maximum extent of the convective envelope. Then we evolved the new fictitious models well above the Bump region. We have taken into account smoothing lengths of $0.1 H_{p}, 0.2 H_{p}, 0.5 H_{p}$, and $0.75 H_{p}$, where $H_{p}$ is the local pressure scale height at the H-discontinuity. For the sake of simplicity the chemical profiles in the smoothing region have been assumed to be linear, and the envelope abundances have been modified in such a way that the sum of element abundances by mass within the structure is perfectly conserved. Current smoothing lengths have been selected to fulfill 
two requirements: i) to avoid a large shift in the luminosity of the Bump along the LF. In fact, predicted and observed $\Delta V_{\text {Bump }}^{H B}$ are, within current observational and theoretical uncertainties, in satisfactory agreement. ii) To introduce a slight change in the sharpness of the H-discontinuity. In fact, the hydrogen distributed in the smoothed region has to be taken from the chemical homogeneous region located above the H-discontinuity. However, substantial changes in the chemical composition (mainly the hydrogen abundance) of this region causes a sizable variation in the star counts across the Bump. Once again current theoretical predictions seem to agree quite well with empirical estimates (Bono et al. 2001).

Figure 1 shows the hydrogen profile ( $\mathrm{H}$ abundance per unit mass) around the lower edge of the convective region at its maximum extent. The dashed line shows the canonical

profile and presents a sharp H-discontinuity, while the solid line shows the H-profile after a linear smoothing of $0.5 H_{p}$ has been applied. The $\mathrm{H}$ abundance in the envelope attains quite similar values in the two cases, and indeed the difference is smaller than 0.001. This means that the jump in the $\mathrm{H}$ abundance between the envelope and the interior is very similar, but in the nonstandard models the H-discontinuity has been smoothed over a thicker region when compared with the standard one.

\section{The effect of the H-discontinuity on the shape of the Bump}

To study in more detail the effect of the sharpness in the H-discontinuity on the shape of the RGB Bump, we computed the RGB luminosity functions of current stellar models. Figure 2 shows the LF of the RGB region located across the Bump for our standard models i.e. $M / M_{\odot}=1, \mathrm{Z}=0.006, \mathrm{Y}=0.23$ constructed by neglecting both atomic diffusion and convective overshooting.

The LF has been computed using a Monte Carlo technique, the number of objects at a given luminosity being proportional to the local evolutionary timescale. We have used an extremely large number of objects to avoid spurious statistical fluctuations in a given luminosity bin. The bin size of the LF plotted in Fig. 2 is 0.02 mag. It is worth mentioning that the intrinsic shape of the Bump is asymmetric and presents a well-defined peak at its brightest end. Note that both the atomic diffusion and the convective overshooting below the formal boundary of the convective envelope do not change the shape of the Bump, since in these models the H-discontinuity is as sharp as in standard models.

According to Bono et al. (2001) we define the parameter $R_{\text {Bump }}$, as the number of stars located within $\pm 0.4 \mathrm{mag}$ above and below the luminosity peak of the Bump, normalized to the number of RGB stars in the region between +0.5 and +1.5 mag below the peak of 
the Bump. The standard models supply $\mathrm{R}_{\mathrm{Bump}}=0.527$, while the models constructed by accounting for atomic diffusion, convective overshooting, or the smoothed hydrogen profile have values within 0.05 of the standard one. This difference is quite negligible, since it is smaller than the typical observational error barl (see Bono et al. 2001). On the other hand, the luminosity peak of the Bump shifts by $\sim 0.07 \mathrm{mag}$ in the models that account for atomic diffusion, (see also Cassisi, Degl'Innocenti \& Salaris 1997), while for the models that include convective overshooting the brightness changes according to the following derivative $\sim 0.8 \mathrm{mag} / H_{p}$. At the same time, we found that an increase of $0.1 H_{p}$ in the smoothing length causes in the nonstandard models a shift of $\sim 0.025$ magnitudes in the position of the Bump. Figure 3 shows the effect of the smoothing on the shape of the Bump. The dashed line refers to the standard LF, while the solid lines display the LFs of smoothed models. They have been plotted by shifting the brightest boundary of the nonstandard Bumps over the standard one. The different LFs are normalized to the same number of stars above the Bump (where the hydrogen profile is the same in all models).

The change in the shape of the Bump as a function of the smoothing length is quite evident. In particular, the Bump becomes more centrally peaked and more symmetric for an increase in the thickness of the smoothing region. For smoothing lengths equal or larger than $0.5 H_{p}$ the shape of the Bump is substantially different than for the standard models. According to the numerical experiments we already performed, such an effect cannot be produced by atomic diffusion nor by convective overshooting. The reason for the difference in the shape of the Bump is outlined in Fig. 4. This figure shows the evolution in the Color-Magnitude-Diagram of our standard models and of the models with the $\mathrm{H}$-discontinuity smoothed over a region of $0.5 \mathrm{H}_{p}$. A glance at the data plotted in this figure clearly shows that the Bump region narrows in the latter case, and this change causes a narrowing of the Bump in the differential LF as well.

The physical explanation of this behavior has to be related to the abrupt change of the mean molecular weight $\mu$ in the region across the chemical discontinuity left over by the outer convection during the first dredge up, and to the strong dependence of the H-burning efficiency on $\mu$. Standard models show a sharp change in the mean molecular weight at the chemical discontinuity; this feature strongly affects the H-burning efficiency, and in turn the stellar surface luminosity. In stellar models whose H-discontinuity has been smoothed, the sharpness of the $\mu$ variation is anticorrelated with the thickness of the smoothing region; as a consequence, the change of the H-burning efficiency is significantly lower in smoothed models than in canonical ones. The top panel of Fig. 5 shows the H-burning luminosity

\footnotetext{
${ }^{1}$ The $R_{\text {Bump }}$ value of the smoothed models is similar to the standard one, since the current smoothing length was chosen to avoid a substantial change in the jump of the H-profile.
} 
produced via CNO cycle as a function of time. Data plotted in this figure disclose that the luminosity drop taking place during the crossing of the H-discontinuity is clearly correlated with the smoothing length.

The smoothing of the H-discontinuity affects not only $\mu$, but also the opacity profile within the structure. To test the hypothesis that the change of opacity may play a role in causing the luminosity drop at the bump location, we performed the following test. We constructed a fictitious model in which the opacity in the region just below the H-discontinuity has been computed by adopting the same chemical composition of the region located above the $\mathrm{H}$-discontinuity. Data plotted in the bottom panel of Fig. 5 clearly show that the change in the opacity profile caused by the variation in the chemical stratification has a negligible effect on the RGB Bump. Therefore, it is the change of $\mu$ which determines the shape of bump region.

At the same time, we have to account for the difference in the evolutionary timescales, since the number of stars in a given magnitude bin is proportional to this quantity. From top to bottom the panels in Fig. 6 show, for the two models plotted in Fig.4, the logarithm of the stellar counts along the three branches of the Bump region as a function of the $\mathrm{V}$ magnitude. The data for the nonstandard models were shifted in brightness as in Fig. 3. The arrows mark the direction during these evolutionary phases, i.e. the change in the surface luminosity. Panel a) shows the star counts before the temporary decrease in luminosity, while panel b) during the subsequent decrease in luminosity. The evolutionary timescale of the standard models at the bin where the luminosity does not increase is roughly a factor of 2 longer than in the smoothed models, while it is systematically shorter during the phases approaching this point and the phases in which the luminosity decreases. During this latter phase the standard models reach the secondary minimum in luminosity quite rapidly when compared with the smoothed one. In fact, the evolutionary timescale of the smoothed models in the fainter luminosity bin is a factor of 3 longer than in the standard models. Panel c) shows the predicted stellar counts during the phases in which the surface luminosity increases once again. The evolutionary timescale of the standard models is essentially constant, while the smoothed models evolve more rapidly until they approach the bright edge of the Bump region.

In this section we have discussed the difference in the LF between standard and smoothed models, now we wish to address the following question: can this difference be detected in actual RGB Bumps? To answer this question we performed new Monte-Carlo simulations, by accounting for different sample sizes and observational errors. Fig. 7 shows the same LFs plotted in Fig. 2, but they were computed by adopting a bin size of 0.05 mag and a $1 \sigma$ random photometric error (the accuracy of the photometric zero point is 
not relevant in this discussion) by $0.025 \mathrm{mag}$. Data plotted in this figure suggest that smoothing lengths equal or larger than $0.5 H_{p}$ still affect the shape of the Bump in such a way that the difference between standard and smoothed models can be detected. According to our simulations, to detect smoothing lengths equal or larger than $0.5 H_{p}$ in metal-rich clusters with metal abundances larger than $[\mathrm{Fe} / \mathrm{H}]=-1$ and not affected by differential reddening, are necessary stellar samples larger than $\sim 120$ stars within \pm 0.2 mag the peak of the Bump, a bin size not larger than 0.06 mag and a $1 \sigma$ random photometric errors not larger than 0.03 mag.

For metallicities equal or lower than $[\mathrm{Fe} / \mathrm{H}]=-1$ the change in the shape of the Bump can be barely detected. Fig. 8 shows the standard RGB LF for $\mathrm{Z}=0.006$ (dotted line) together with the RGB LF obtained from a $1 M_{\odot}$ standard models (no diffusion, no overshooting) with $\mathrm{Z}=0.002$ (solid line). By assuming an $\alpha$-enhancement $[\alpha / \mathrm{Fe}]=0.4$ the latter metallicity becomes $[\mathrm{Fe} / \mathrm{H}]=-1$. The dashed line refers to the RGB LF of evolutionary models constructed by adopting the same mass value and metallicity $\left(1 M_{\odot}\right.$, $\mathrm{Z}=0.002$ ) but with the H-discontinuity smoothed over $0.5 H_{p}$. The LFs of the less metal-rich models have been shifted by 0.5 mag to match the bright edge of the Bump region of the LF for $\mathrm{Z}=0.006$.

Even though the main effect of the smoothing is qualitatively similar to the case of more metal-rich models, the extent of the Bump for $\mathrm{Z}=0.002$ is smaller both in the standard and in smoothed models. This means that smaller photometric errors, smaller bin sizes, and larger samples of RGB stars are mandatory to detect a change in the shape of the Bump. This result seems in contrast with the finding brought out by Bono et al. (2001) that the value of the $R_{\text {Bump }}$ parameter is almost constant with metallicity. However, the contradiction is only apparent, and indeed toward lower metallicities the Bump becomes brighter, and therefore the number of stars in the normalization region decreases as well. This effect makes the $\mathrm{R}_{\text {Bump }}$ value only marginally dependent on the cluster metallicity.

\section{Conclusions and final remarks}

We investigated in detail the dependence of both shape and location of the Bump along the RGB LF on the smoothing of the H-discontinuity at the base of the convective envelope in Population II structures. The reason to account for this smoothing is related to the possible occurrence of partial mixing processes below the lower boundary of the convective region. Current spectroscopic measurements of RGB stars are somehow at odds with theoretical predictions. To account for the low carbon isotopic ratios and for the $\mathrm{Li}$ abundance in population II stars it has been suggested that rotation-induced mixing 
occurs above the RGB Bump (Sweigart \& Mengel 1979; Charbonnel 1995). However, even by accounting for this extra-mixing process the agreement between empirical data and theory is far from being satisfactory (Thévenin et al. 2001). The occurrence of mixing below the bottom of the convective region has been generally neglected, since theoretical predictions (Mestel 1957) and spectroscopic data (Charbonnel, Brown, \& Wallerstein 1998, and references therein) suggest that the occurrence of gradients in the molecular weight, such as the H-discontinuity in the envelope of RGB stars, stabilizes the mixing processes induced by rotation. However, current spectroscopic measurements support the evidence that RGB stars located across the Bump present large Li abundances (Charbonnel \& Balachandran 2000). This suggests that these stars underwent Li production soon before or at the Bump phase. The comparison between predicted and observed shape of RGB Bumps can supply tight constraints on the sharpness of the H-discontinuity, and in turn on the inhibiting effect of molecular weight barriers on extra-mixing related either to rotation or to exotic phenomena.

We constructed several stellar models by assuming different smoothing lengths, and we found that for smoothings up to $0.75 H_{p}$ current theoretical predictions concerning the difference in luminosity between the peak of RGB Bump and the HB, $\Delta V_{B u m p}^{H B}$, and the star counts across the Bump region, $R_{\text {Bump }}$, are still in satisfactory agreement with observations. However, as expected, a change in the H-abundance profile significantly affects the evolutionary timescales of the H-burning shell, and in turn the shape of the RGB Bump along the LF. Interestingly enough, we found that it is possible to constrain the sharpness of the H-discontinuity at the base of the envelope of RGB stars by comparing predicted and empirical Bump shapes of differential LFs in metal-rich GGCs.

In this context it is worth mentioning that this finding is far from being a speculative issue. In fact, the new Advanced Camera for Surveys (ACS, Clampin et al. 2000) on board of HST will supply, thanks to its wide field of view $\left(202 \times 202 \operatorname{arcsec}^{2}\right)$ and homogeneous spatial resolution $(0.05 \mathrm{arcsec} / \mathrm{pixel})$, a complete census of RGB stars over a substantial fraction of GGCs innermost regions. In particular, for clusters characterized by relatively high central densities, the ACS can measure at the star-by-star level the bulk of cluster RGB stars. As a consequence, the detection of the shape of RGB bump among massive metal-rich clusters will become feasible in the near future. To constrain on a quantitative basis the empirical requirements necessary to supply an accurate detection of the shape of the Bump. we performed several Monte-Carlo experiments according to current theoretical predictions. We found that a robust detection requires a minimum sample of more than $\sim 120$ RGB stars within \pm 0.2 mag the peak of the Bump, the use of a bin size up to 0.06 mag in the LF, as well as random photometric errors smaller than 0.03 mag. Fortunately enough, several metal-rich GGCs and the new scientific capabilities of HST can allow us to 
cope with this challenging and intriguing project.

We are grateful to an anonymous referee for useful suggestions that improved the content of an early draft of the paper. M.S. wishes to acknowledge the Osservatorio Astronomico di Collurania and the Max Planck Institut für Astrophysik for their kind hospitality during the completion of this work. We warmly thank M. Zoccali for several interesting discussions on HST photometry and for useful comments on early draft of this paper. This work was supported by MURST/Cofin2000 under the project: "Stellar Observables of cosmological relevance" (S. C. \& G. B.). 


\section{REFERENCES}

Alongi , M., Bertelli, G., Bressan, A., \& Chiosi, C. 1991, A\&A, 244, 95

Alves, D. R., \& Sarajedini, A. 1999, ApJ, 511, 225

Bergbusch, P. A., \& Vandenberg, D. A. 2001, ApJ, 556, 322

Bono, G., Cassisi, S., Zoccali, M., \& Piotto, G. 2001, ApJ, 546, L109

Bono, G., \& Castellani, V. 1992, A\&A, 258, 385

Cassisi, S., Degl'Innocenti, S., \& Salaris, M. 1997, MNRAS, 290, 515

Cassisi, S., \& Salaris, M. 1997, MNRAS, 285, 593

Castelli, F., Gratton, R. G., \& Kurucz, R. L. 1997a, A\&A, 318, 841

Castelli, F., Gratton, R. G., \& Kurucz, R. L. 1997b, A\&A, 324, 432

Charbonnel, C. 1995, ApJ, 453, L41

Charbonnel, C., \& Balachandran, S.C. 2000, A\&A, 359, 563

Charbonnel, C., Brown, J. A., \& Wallerstein, G. 1998, A\&A, 332, 204

Clampin, M., \& ACS Science Team 2000, BAAS, 197, 1218

Ferraro, F. R., Messineo, M., Fusi Pecci, F., de Palo, M. A., Straniero, O., Chieffi, A., \& Limongi, M. 1999, ApJ, 118, 1738

Fusi Pecci, F., Ferraro, F. R., Crocker, D. A., Rood, R. T., \& Buonanno, R. 1990, A\&A, 238, 95

Gratton, R. G., et al. 2001, A\&A, 369, 87

Iben, I., Jr. 1968, ApJ, 154, 581

King, C. R., Da Costa, G. S., \& Demarque, P. 1985, ApJ, 299, 674

Mestel, L. 1957, ApJ, 126, 550

Rutledge, G. A., Hesser, J. E., \& Stetson, P. B. 1997, AJ, 109, 907

Sweigart, A. V., \& Mengel, J. G. 1979, ApJ, 229, 624 
Thévenin, F., Charbonnel, C., de Freitas Pacheco, J. A., Idiart, T. P., Jasniewicz, G., de Laverny, P., \& Plez, B. 2001, A\&A, 373, 905

Thomas, H. C. 1967, Ph.D. thesis, L. M. Univ., Münich

Zoccali, M., Cassisi, S., Piotto, G., Bono, G., \& Salaris, M. 1999, ApJ, 518, L49

Zoccali, M., \& Piotto, G. 2000, A\&A, 358, 943 
Fig. 1.- Hydrogen profile (abundance by mass fraction) as a function of the stellar mass fraction. The dashed line shows the H-profile of our standard models (no atomic diffusion, no convective overshooting) constructed by adopting $M / M_{\odot}=1.0, \mathrm{Y}=0.23$, and $\mathrm{Z}=0.006$, while the solid line refers to models constructed with same input parameters but the $\mathrm{H}$ discontinuity was smoothed out over a region whose thickness is $0.5 H_{p}$. See text for more details.

Fig. 2.- Red giant branch differential LF, i.e. logarithm of star counts, as a function of $M_{V}$ across the Bump region of the standard models. The bin size is of 0.02 mag.

Fig. 3.- Comparison between the differential LF of our standard models (dotted line) and the LFs of the models constructed by artificially smoothing the H-discontinuity over a region of $0.1 H_{p}, 0.2 H_{p}, 0.5 H_{p}, 0.75 H_{p}$ (solid lines). The LFs of the smoothed models have been shifted in luminosity to match the bright edge of the Bump of the standard models.

Fig. 4.- Color-Magnitude-Diagram of the RGB Bump phase for the standard models (dotted line) and for the models with the H-discontinuity smoothed out over a region of $0.5 H_{p}$ (solid line).

Fig. 5.- Top: Time behavior of the H-burning luminosity produced via CNO cycle during the RGB Bump phase for our standard model and models with artificially smoothed $\mathrm{H}-$ discontinuity. Bottom: Time behavior of the stellar surface luminosity during the RGB Bump phase for a standard model (solid line), a model constructed by smoothing the $\mathrm{H}-$ discontinuity over a region of $0.5 H_{p}$ (long dashed line), and a standard model computed by artificially changing the opacity in the region across the H-discontinuity (dotted line, see text for more details).

Fig. 6.- Comparison between the differential LFs of the standard models (dotted line) and of the models whose H-discontinuity whose smoothed out over a region of $0.5 H_{p}$ (solid line). From top to bottom the three panels refer to different evolutionary phases across the bump, namely a) from the faint edge of the Bump to the secondary maximum, b) from the secondary maximum to the secondary minimum, and c) from the secondary minimum to the bright edge of the Bump. The arrows mark the evolutionary direction, i.e. the change in the surface luminosity along the three branches.

Fig. 7. - Same as in Fig. 3, but the differential LF was constructed by adopting a bin size of $0.05 \mathrm{mag}$ and $1 \sigma$ random photometric errors of $0.025 \mathrm{mag}$.

Fig. 8. - Comparison between different differential LFs constructed by adopting the same stellar mass $-M / M_{\odot}=1.0$ - but different chemical compositions. The dotted and the solid 
line show the LFs of the standard models constructed by adopting $\mathrm{Z}=0.006$ and $\mathrm{Z}=0.002$ respectively. The dashed line displays the $\mathrm{LF}$ of the model with $\mathrm{Z}=0.002$ and the $\mathrm{H}$ discontinuity smoothed out over a region of $0.5 H_{p}$. The bin size is of $0.02 \mathrm{mag}$. The LFs for $\mathrm{Z}=0.002$ have been shifted in luminosity to match the bright edge of the Bump. 


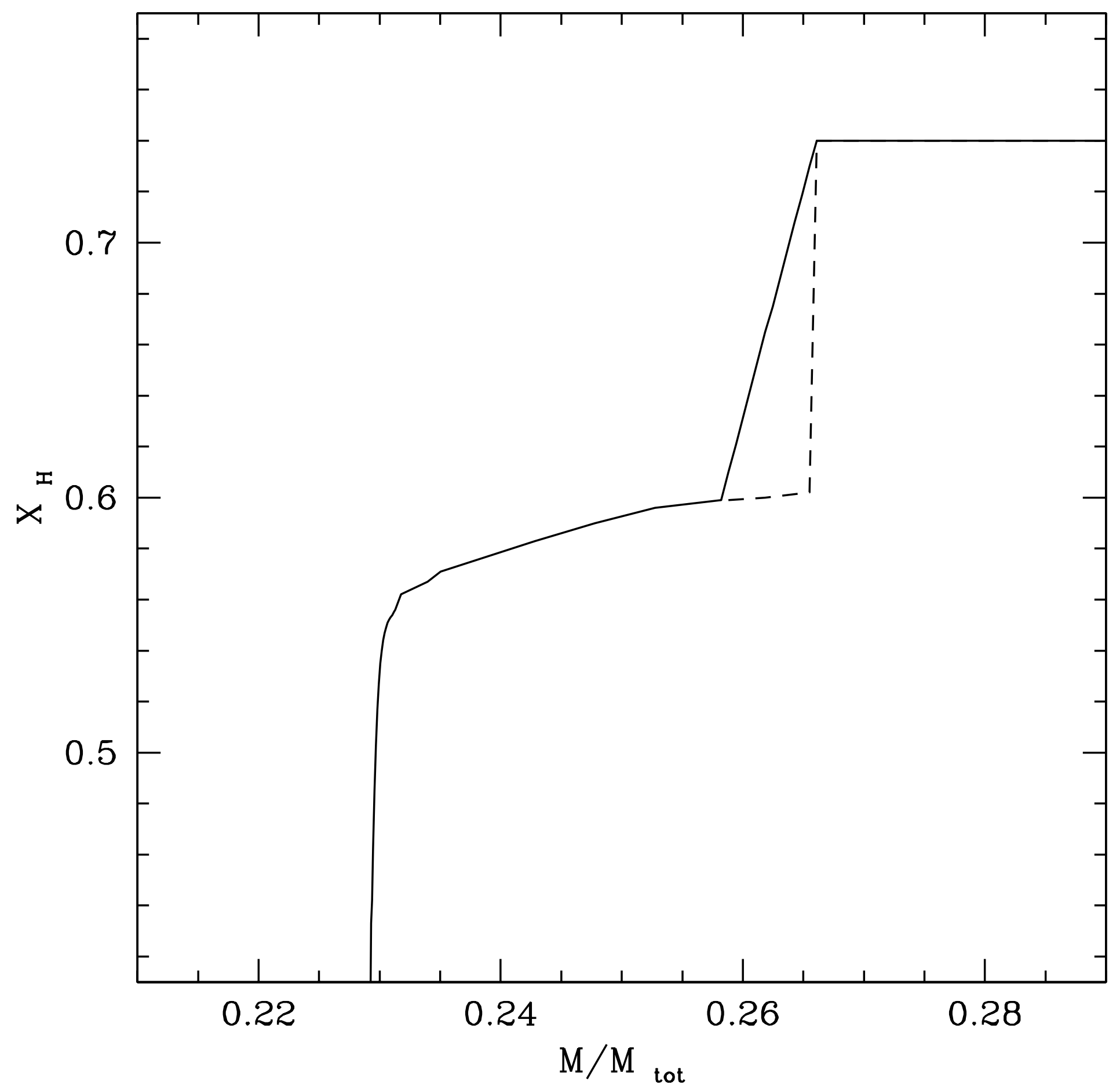




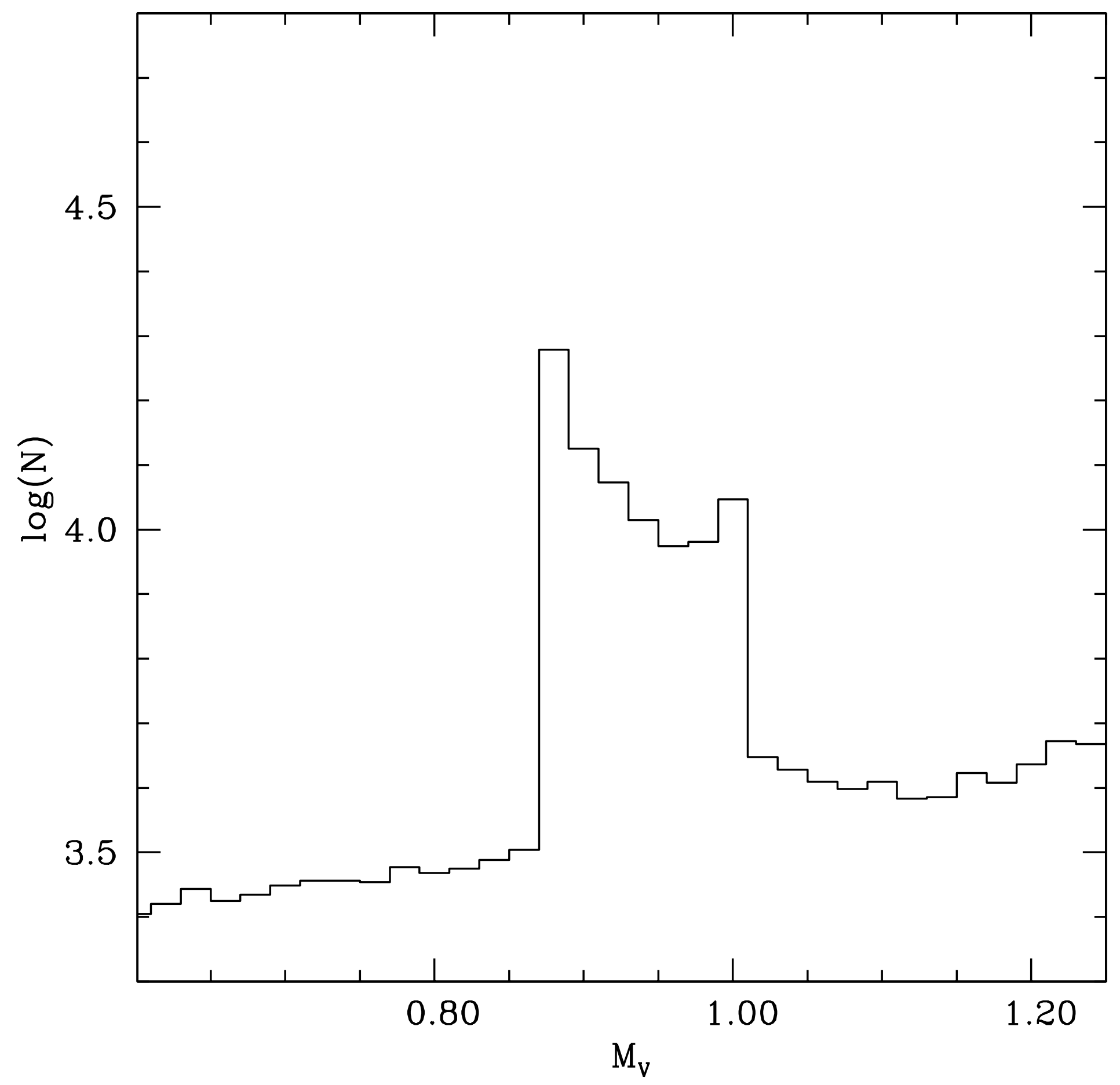




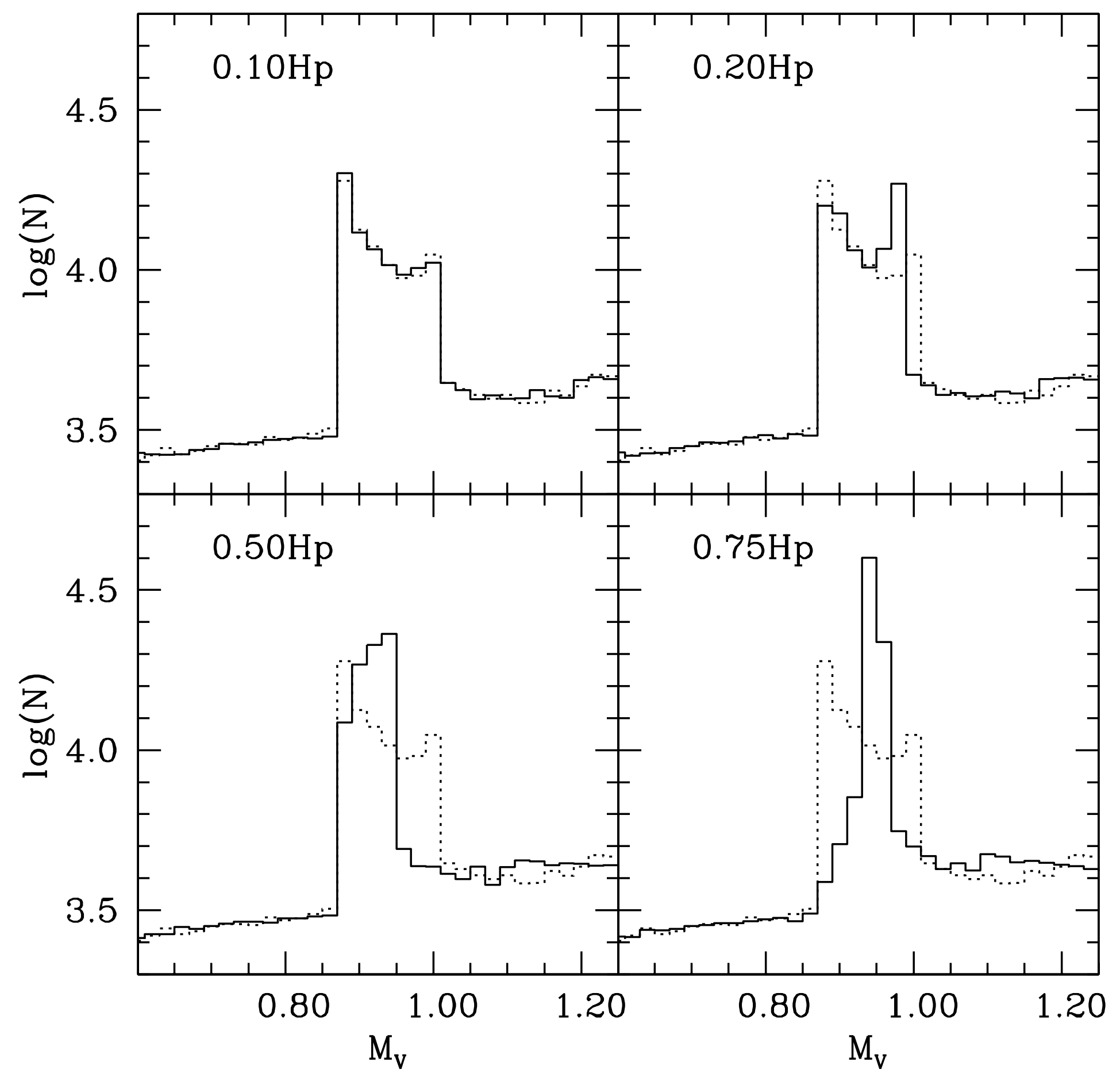




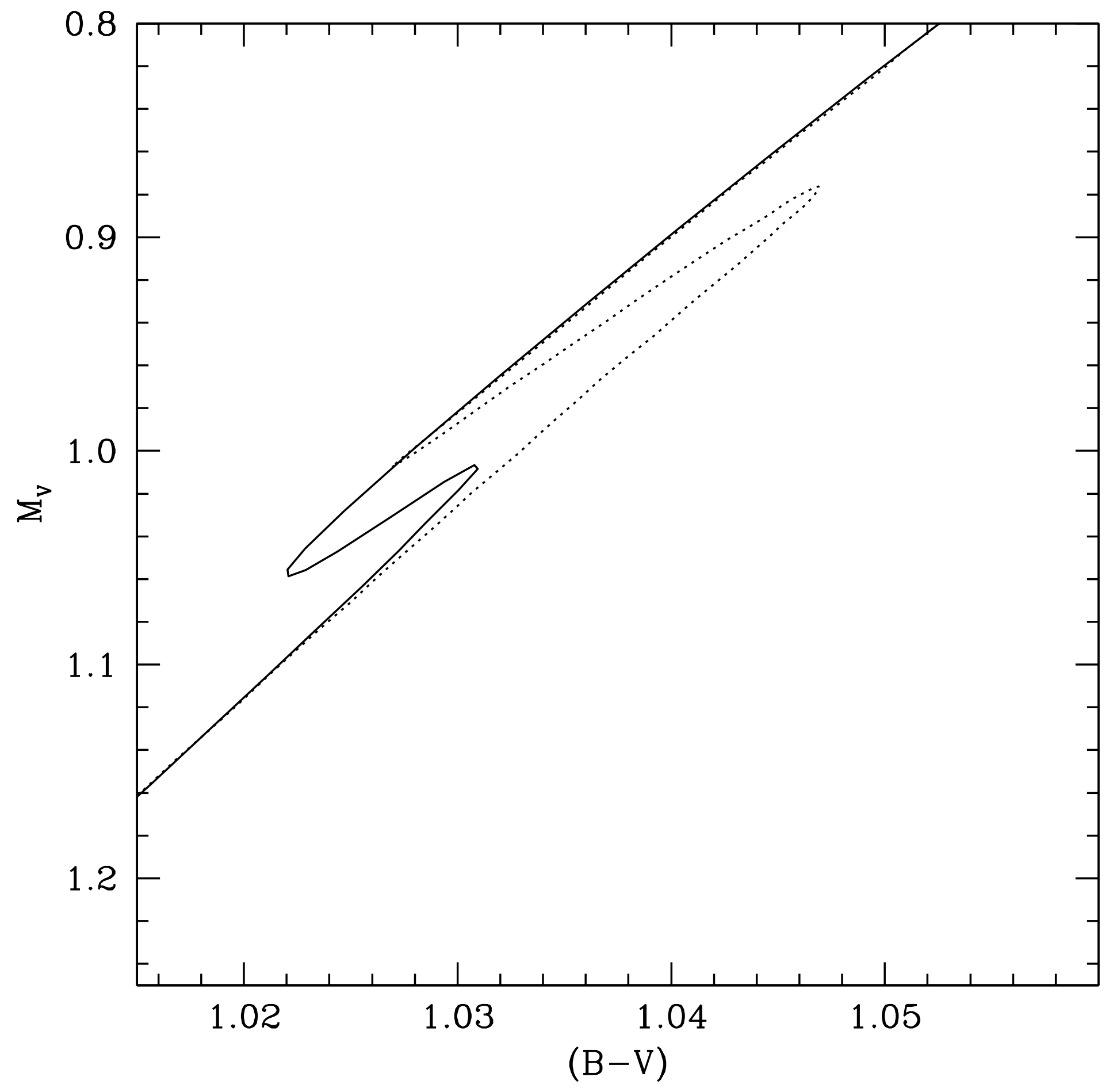




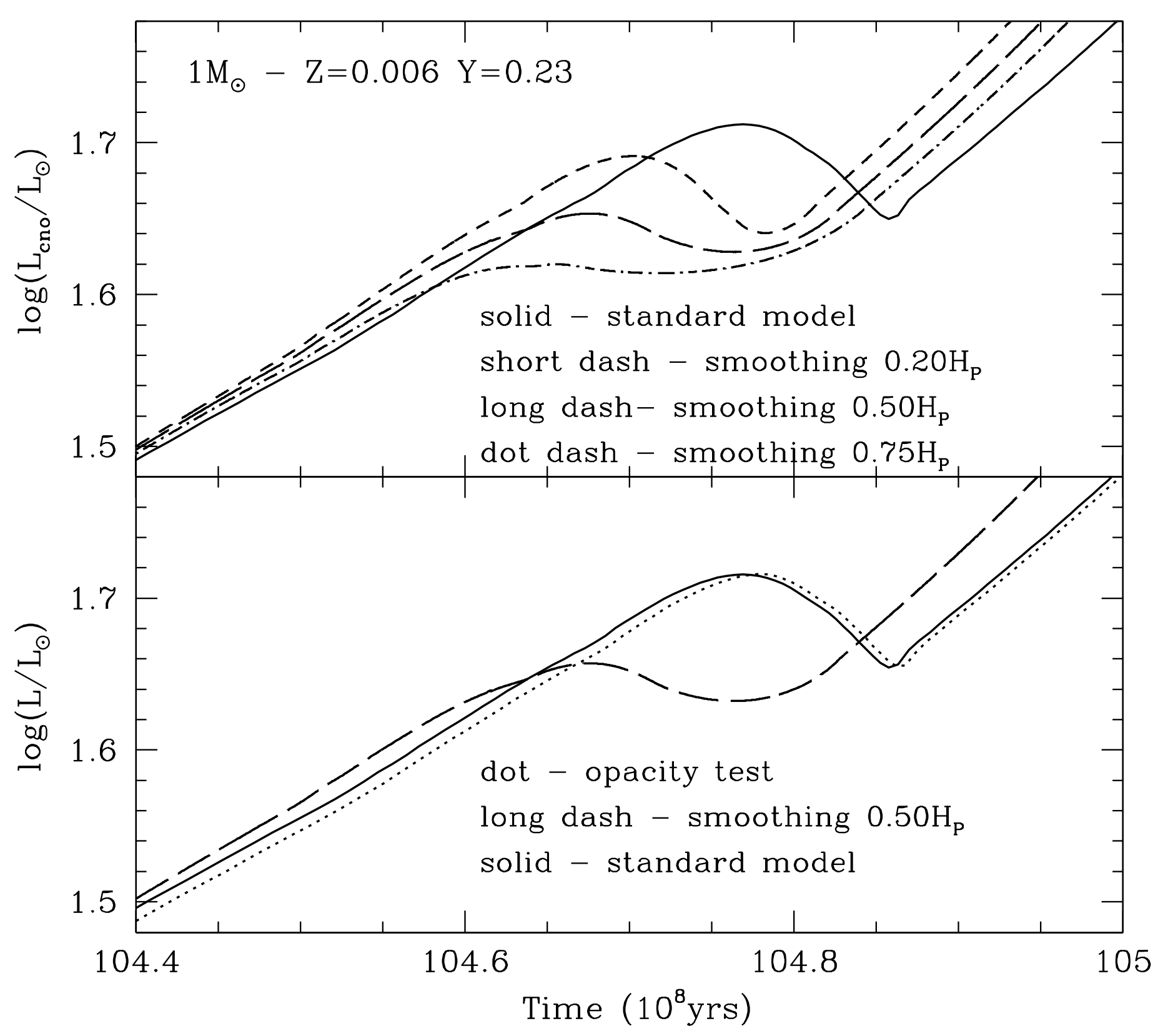




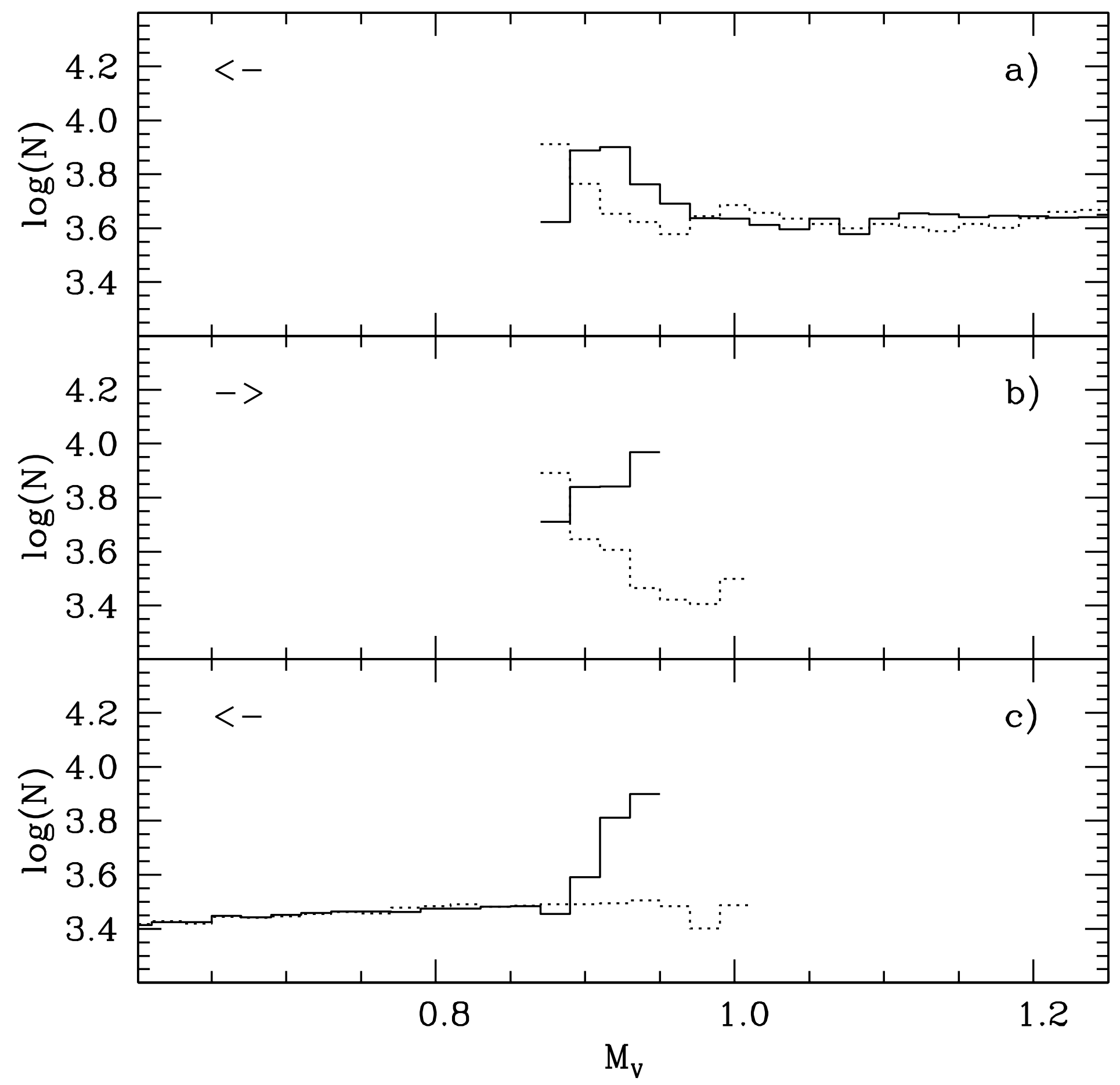




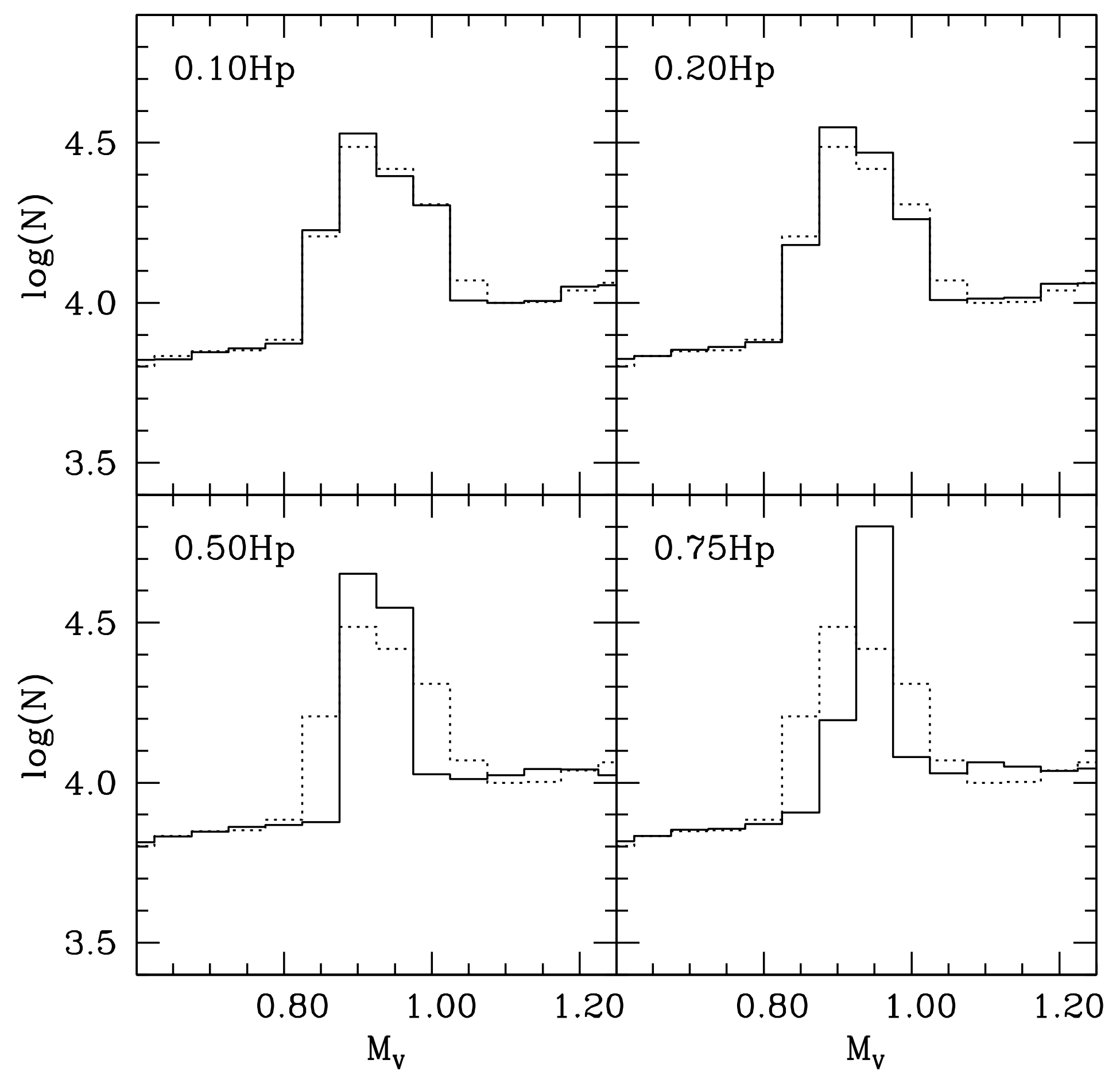




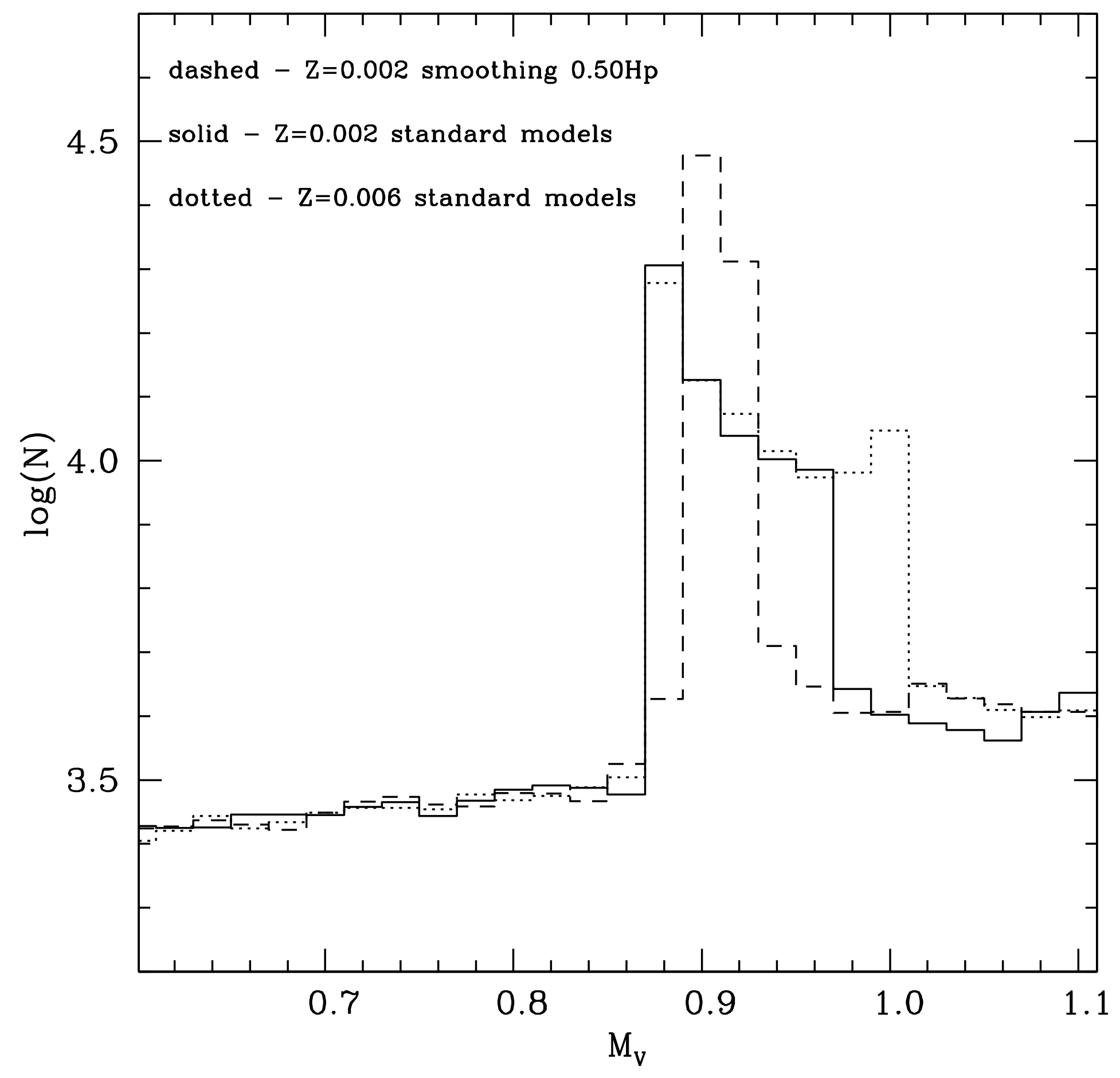

